\title{
Effect of Circuit Parameters on Stability of Voltage-fed Buck-Boost Converter in Discontinuous Conduction Mode
}

\author{
Zhao-He Feng*, Ren-Xi Gong ${ }^{\dagger}$ and Qing-Yu Wang*
}

\begin{abstract}
The state transition matrix are obtained by solving state equations in terms of Laplace inverse transformation and Cayley-Hamilton theorem, and an establishment of a precise discreteiterative mapping of the voltage-fed buck-boost converter operating in discontinuous conduction mode is made. On the basis of the mapping, the converter bifurcation diagrams and Lyapunov exponent diagrams with the input voltage, the resistance, the inductance and the capacitance as the bifurcation parameters are obtained, and the effect of the parameters on the system stability is deeply studied. The results obtained show that they have a great influence on the stability of the system, and the general trend is that the increase of either the voltage-fed coefficient, input voltage or the load resistance, or the decrease of the filtering inductance, capacitance will make the system stability become poorer, and that all the parameters have a critical value, and when they are greater or less than the values, the system will go through stable $1 \mathrm{~T}$ orbits, stable $2 \mathrm{~T}$ orbits, $4 \mathrm{~T}$ orbits, $8 \mathrm{~T}$ orbits and eventually approaches chaos.
\end{abstract}

Keywords: Buck-Boost converter, Precise discrete-iterative map, Bifurcation, Lyapunov exponent, Stability

\section{Introduction}

DC/DC power converters have found wide applications in industrial automation, power electronics, communications and many other fields. With them operating, some irregular phenomena often arise, such as a sudden collapse of the running state, an unknown electromagnetic noise, instability of the running system. These behaviors greatly affect the stability of the system [1-4]. However, they have been often ignored or treated as a fault by practical circuit designers for a long time. A buck-boost converter, as an important kind of DC/DC converter, has been widely used because of its wide range of input voltage, and advantages with both buck and boost converters [5, 6]. However, a variety of nonlinear behaviors, such as sub harmonics, bifurcation and chaos, are easily caused due to the cyclic switching of the circuit topologies [7-9]. Up to now, their studies are mainly focused on modeling or analyzing the nonlinear phenomena [10-12]. The methods adopted can be approximately divided into two kinds: numerical method and approximate model analytical method. In the former, the equations characterizing nonlinear behaviors of converters are solving by numerical iterative algorithms [13]. In the latter, the analytical solutions are obtained by making some approximate disposal [14]. Because of the discrete solutions obtained in numerical method, it is not readily to understand the dependence

$\dagger$ Corresponding Author: School of Electrical Engineering, Guangxi University, Nanning 530004, China. (rxgong@gxu.edu.cn)

* School of Electrical Engineering, Guangxi University, Nanning 530004, China. (f_zhaohe@163.com,wangqy881018@sina.com)

Received: June 23, 2013; Accepted: January 25, 2014 of the circuit parameters on the stability of converters clearly. Although the analytical solutions can be achieved by the approximate model analytical method, some useful information about these systems is ignored. Subtle differences in the parameters can lead to very different results and the truth of conclusion will be questioned, especially for the nonlinear system. Therefore, it is very necessary to establish a precise discreteiterative mapping. In this paper, precise discrete mapping which operates in the discontinuous conduction mode (DCM) has been established by a series of mathematical deduction without any approximation. Based on the model, the influence of the circuit parameters on the stability of the converter is analyzed.

\section{Precise Discrete Mapping for the Buck-Boost Converter}

The circuit model of a buck-boost converter is shown in Fig. 1, in which a switch $S$, a diode $V D$, a capacitor $C$, an inductor $L$, a load resistor $R$, an input voltage source $E$ and a control-loop are included. The control-loop comprises two comparators and an amplifier, of which the given values of the comparators are respectively the desired output voltage $\mathrm{V}_{\mathrm{o}}$ and the duty cycle $\mathrm{D}$ in steady-state, and the gain of the amplifier is voltage-fed coefficient $k$. In the model, the equivalent-series-resistance $r_{\mathrm{c}}$ of $C$ is considered. Obviously, the circuit is of second-order. There are three kinds of switch states in DCM: (1) $S$ on and $V D$ off; (2) $S$ off and $V D$ on; (3) $S$ and $V D$ off. 


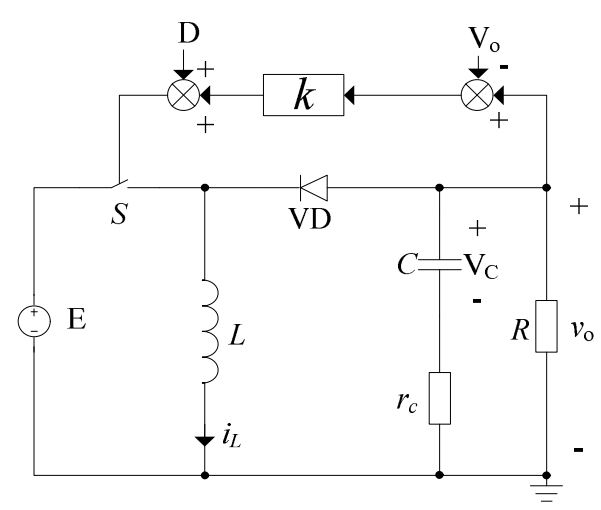

Fig. 1. Circuit model of voltage mode controlled buckboost converter

The converter can be described by the following sequence of state equations

$$
\begin{cases}\dot{x}=A_{1} x+B_{1} E & t_{n} \leq x<t_{n}^{\prime} \\ \dot{x}=A_{2} x+B_{2} E & t_{n}^{\prime} \leq x<t_{n}^{\prime} \\ \dot{x}=A_{3} x+B_{3} E & t_{n}^{\prime} \leq x<t_{n+1}\end{cases}
$$

where

$$
\begin{gathered}
A_{1}=\frac{1}{C\left(R+r_{c}\right)}\left[\begin{array}{cc}
-1 & 0 \\
0 & 0
\end{array}\right], B_{2}=\left[\begin{array}{l}
0 \\
\frac{1}{L}
\end{array}\right] \\
A_{2}=\frac{1}{C\left(R+r_{c}\right)}\left[\begin{array}{cc}
-1 & R \\
-\frac{C R}{L} & -\frac{C R r_{c}}{L}
\end{array}\right], B_{2}=\left[\begin{array}{l}
0 \\
0
\end{array}\right] \\
A_{3}=\frac{1}{C\left(R+r_{c}\right)}\left[\begin{array}{cc}
-1 & 0 \\
0 & 0
\end{array}\right], B_{2}=\left[\begin{array}{l}
0 \\
0
\end{array}\right]
\end{gathered}
$$

Where, $x$ is the state vector of the circuit, i.e., $x=\left[\mathrm{V}_{\mathrm{C}} i_{\mathrm{L}}\right]^{\mathrm{T}}$, subscript $n$ denotes the value at the beginning of the $n$th cycle, $T$ is the switching period, and $t_{c}, t_{d}$ and $t_{e}$ are the running times in different stages of an switching period, which are given by:

$$
\begin{gathered}
t_{c}=t_{n}^{\prime}-t_{n}, t_{d}=t_{n}^{\prime \prime}-t_{n}^{\prime}, t_{e}=t_{n+1}-t_{n}^{\prime \prime} \\
\left\{\begin{array}{l}
t_{c}=d_{n} T \\
t_{d}=\frac{E}{V_{c}\left(t_{n}\right)} \cdot t_{c} \\
t_{e}=T-t_{c}-t_{d}
\end{array}\right.
\end{gathered}
$$

Where $d_{n}$ is the duty cycle during the $n$th period, defined as

$$
d_{n}=D-k\left[V_{c}\left(t_{n}\right)-V_{o}\right]
$$

Using a successive iterative method, the value of $x_{\mathrm{n}+1}$ can be expressed in terms of $x_{n}$.

$$
\begin{aligned}
x_{n+1}= & e^{A_{3} t_{e}} e^{A_{2} t_{d}} e^{A_{1} t_{c}}\left[x_{n}+\int_{t_{n}}^{t_{n}^{\prime}} e^{A_{1}\left(t_{n}-\tau\right)} B_{1} E d \tau\right]+ \\
& e^{A_{3} t_{e}} e^{A_{2} t_{d}} \int_{t_{n}^{\prime}}^{t_{n}^{\prime \prime}} e^{A_{2}\left(t_{n}^{\prime}-\tau\right)} B_{2} E d \tau+ \\
& e^{A_{3} t_{e}} \int_{t_{n}^{\prime \prime}}^{t_{n+1}} e^{A_{3}\left(t_{n}^{\prime \prime}-\tau\right)} B_{3} E d \tau
\end{aligned}
$$

Where, $e^{A_{i} t}$ is the transition matrix corresponding to $A_{i}(i=1,2,3)$, and they affect the accuracy of the discrete model.

The state transition matrixes are obtained by solving Eq. (1) in terms of Laplace inverse transformation and CayleyHamilton theorem,

$$
\begin{gathered}
e^{A_{1} t}=e^{A_{3} t}=\left[\begin{array}{cc}
e^{-m t} & 0 \\
0 & 1
\end{array}\right] \\
e^{A_{2} t}=a_{0} I+a_{1} A_{2}=\left[\begin{array}{cc}
a_{0}-m a_{1} & R m a_{1} \\
-\frac{C R}{L} m a_{1} & a_{0}-\frac{C R r_{c}}{L} m a_{1}
\end{array}\right]
\end{gathered}
$$

where

$$
\begin{gathered}
m=1 / C\left(R+r_{c}\right) \\
a_{0}=\frac{e^{-\alpha m t}}{\beta}[\alpha \sin (\beta m t)+\beta \cos (\beta m t)] \\
a_{1}=\frac{e^{-\alpha m t}}{\beta m} \sin (\beta m t) \\
\alpha=\frac{1}{2}\left[1+\frac{C R r_{c}}{L}\right] \\
\beta=\frac{1}{2} \sqrt{\frac{4 R}{L m}-\left[1+\frac{C R r_{c}}{L}\right]^{2}}
\end{gathered}
$$

and $I$ is a second-order identity matrix.

Using Eqs. (2, 3) and (4), Eq. (8) can be simplified as

$$
x_{n+1}=e^{A_{3} t_{e}} e^{A_{2} t_{d}} e^{A_{1} t_{c}} x_{n}+e^{A_{3} t_{e}} e^{A_{2} t_{d}} \int_{t_{n}}^{t_{n}^{\prime}} e^{A_{1}\left(t_{n}^{\prime}-\tau\right)} B_{1} E d \tau
$$

The precise discrete mapping of the voltage-fed buckboost converter operating in DCM can be obtained by combining Eq. (9) with Eq. (10), and Eq. (16) can be written as

$$
x_{n+1}=e^{-m\left(t_{c}+t_{e}\right)}\left(a_{0}-m a_{1}\right) x_{n}+e^{-m t_{e}} \frac{R E m a_{1} t_{c}}{L}
$$

In DCM of operation, the inductance current $i_{\mathrm{L}}$ is zero at the beginning of every switching cycle, that is, $i_{L}\left(t_{n}\right)=$ $i_{L}(n T)=0$, and only the capacitance functions. Therefore, the iterative mapping is changed as first-order precise discrete mapping.

In the ideal situation, a buck-boost converter can be 
considered as a piecewise-linear circuit, but it constitutes a nonlinear system with voltage feedback incorporated. When the circuit parameters and topology are changed, the stability of the system will be affected, and bifurcation and chaotic behavior may be presented.

A usual way to determine the stability of a fixed point $\mathrm{X}$ is to consider the Taylor series expansion of $\Delta x_{n+1}$ around the fixed point

$$
\Delta x_{n+1}=\left.\sum_{k=1}^{\infty} \frac{1}{k !} \frac{\partial^{k} f(x)}{\partial x^{k}}\right|_{x=X}\left(\Delta x_{n}\right)^{k}
$$

where $\Delta x_{n+1}=x_{n}-X$.

If the disturbance $-\Delta x_{n}$ is small, the higher order terms in Eq. (18) can be negligible. Thus, the stability criterion of DC/DC converter can be simplified as

$$
\left|\frac{\partial x_{n+1}}{\partial x_{n}}\right|=\left|\frac{\partial f(x)}{\partial x}\right|_{x=X} \mid \leq 1
$$

When the stability criterion can not be satisfied, the circuit will eventually evolve into chaos.

On the other hand, Eq. (17) can be represented as by a little transformation

$$
x_{n+1}=\eta_{1} x_{n}+\eta_{2}
$$

where

$$
\begin{gathered}
\eta_{1}=e^{-m\left(t_{c}+t_{e}\right)}\left(a_{0}-m a_{1}\right) x_{n} \\
\eta_{2}=e^{-m t_{e}} \frac{R E m a_{1} t_{c}}{L}
\end{gathered}
$$

Taking the derivative of Eq. (20), gives

$$
\frac{d x_{n+1}}{d x_{n}}=\eta_{1}+\eta_{1}^{\prime} x_{n}+\eta_{2}^{\prime}
$$

where

$$
\begin{gathered}
\eta_{1}^{\prime}=\frac{m E T}{\beta x_{n}} e^{-m\left(t_{c}+t_{e}+\alpha t_{d}\right)}\left(\frac{d_{n}}{x_{n}}+k\right) . \\
\left((1-\alpha)^{2}+\beta^{2}\right) \sin \left(\beta m t_{d}\right) \\
\eta_{2}^{\prime}=\frac{R E}{\beta L} e^{-m\left(t_{e}+\alpha t_{d}\right)}\left[\beta m t_{d}^{\prime} t_{c} \cos \left(\beta m t_{d}\right)+\right. \\
\left.\left(m t_{c}\left(t_{c}^{\prime}+t_{d}^{\prime}\right)-\alpha m t_{c} t_{d}^{\prime}+t_{c}^{\prime}\right) \cdot \sin \left(\beta m t_{d}\right)\right]
\end{gathered}
$$

\section{Stability Analysis of Buck-Boost Converter}

On the basis of the model above, an analysis of the effect of all the main circuit parameters on the stability of a buck- boost converter operating in DCM is made.

\subsection{Effect of voltage-fed coefficient $k$ on system stability}

When the voltage-fed coefficient $k$ is chosen as the bifurcation parameter, the stability criterion can be expressed as Eq. (26) in terms of Eq. (23)

$$
\left|\frac{d x_{n+1}}{d x_{n}}\right|=|-11.4001 k+0.7887| \leq 1
$$

The circuit parameters are given as follows: $T=333.33 \mu \mathrm{s}$, $E=16 \mathrm{~V}, L=208 \mu \mathrm{H}, C=222 \mu \mathrm{F}, \mathrm{V}_{\mathrm{o}}=25 \mathrm{~V}, R=12.5 \Omega$ and $T /\left(C\left(R+r_{c}\right)\right)=0.12$. From Eq. (26), the critical value $k_{c}$ of $k$ can be solved as 0.1569 , and the bifurcation diagram can be obtained as shown in Fig. 2. It can be seen that when $k_{\mathrm{c}}<0.1569$, the buck-boost converter is in the stable state $1 \mathrm{~T}$ regions, but when $k>0.1569$, it is firstly in the stable $2 \mathrm{~T}$ and $4 \mathrm{~T}$, and then stable $8 \mathrm{~T}$, and eventually approaches chaos, whose Lyapunov exponent diagram is shown in Fig. 3 , of which, the positive values on the Lyapunov exponent diagram mean chaos, and at the point of tangential bifurcation, the Lyapunov exponent is less than zero, and the simulation result is well agreement with bifurcation diagram. Actually, tangential bifurcation triggers intermittent chaos. In Fig. 4 is shown the phase portrait around the critical of $k$.

\subsection{Effect of input voltage $E$ on system stability}

Due to its wide input voltage range, a buck-boost

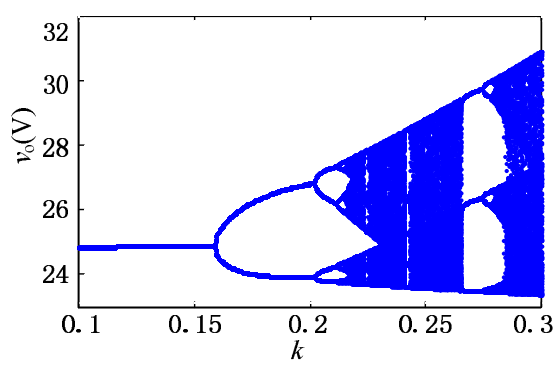

Fig. 2. Bifurcation diagram with the variation of $k$

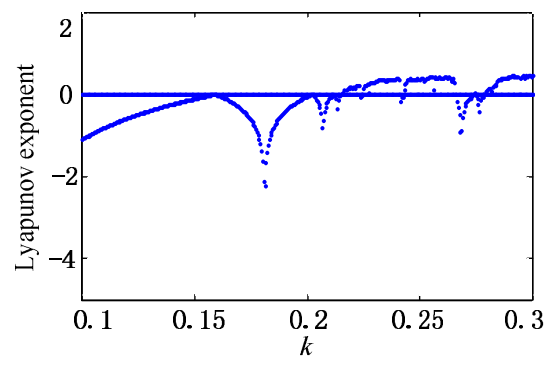

Fig. 3. Lyapunov exponent with the variation of $k$ 


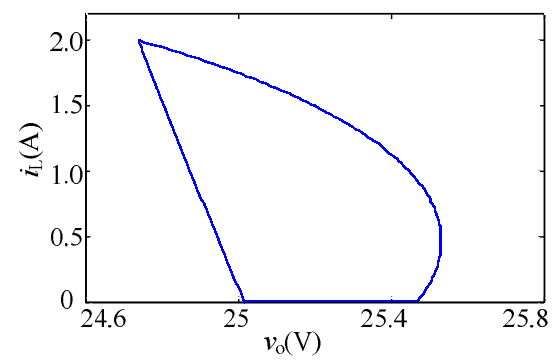

Fig. 4. Phase portraits of sampled $i_{\mathrm{L}}$ versus $v_{\mathrm{o}}$ for $k=0.1569$.

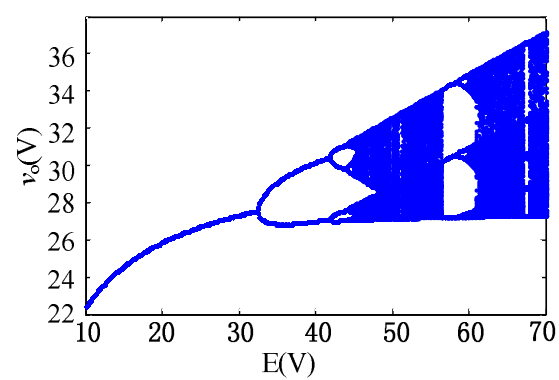

Fig. 5. Bifurcation diagram with the variation of $E$

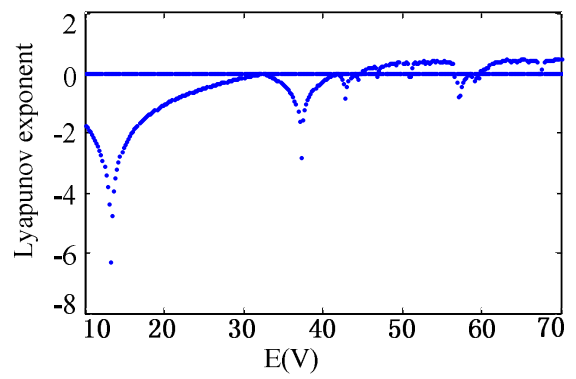

Fig. 6. Variation of Lyapunov exponent with $E$

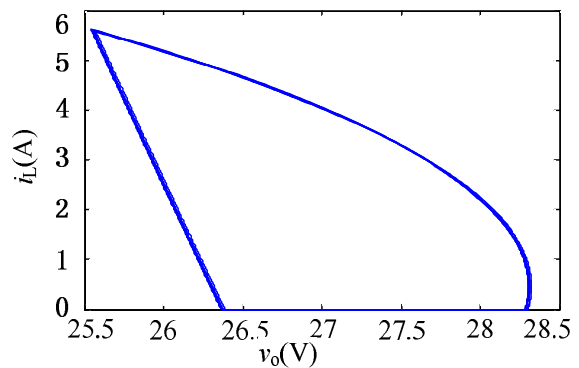

Fig. 7. Phase portraits of sampled $i_{\mathrm{L}}$ versus $v_{\mathrm{o}}$ for $E=32.2 \mathrm{~V}$.

converter has been widely used. Theoretically, a stable output voltage can be obtained by the pulse width modulation (PWM) control, but in fact, the system can remain stable only when the input voltage is in a certain range. In order to study the effect of the input voltage $E$ on the stability of the converter, the bifurcation diagram and Lyapunov exponent diagram with $E$ as bifurcation parameter can be obtained under the condition of $T=$ $333.33 \mu \mathrm{s}, L=208 \mu \mathrm{H}, C=222 \mu \mathrm{F}, \mathrm{V}_{\mathrm{o}}=25 \mathrm{~V}, R=12.5 \Omega$ and $k=0.08$, which are respectively shown in Figs. 5 and Fig. 6 . It can been seen that the critical stable value of $E$ is approximately $32.2 \mathrm{~V}$, that is, the system is stable when $E \leq 32.2 \mathrm{~V}$, and when $E$ is greater than the value, it will go through stable $1 \mathrm{~T}$ orbits, stable $2 \mathrm{~T}$ orbits, $4 \mathrm{~T}$ orbits, $8 \mathrm{~T}$ orbits as $E$ increases, and eventually approaches chaos. In Fig. 7 is shown the phase portrait around the critical of $E$.

\subsection{Effect of inductance $L$ on system stability}

Inductance $L$ is one of the most important parameters determining the circuit operation modes, namely, DCM or continuous conduction mode (CCM). Its critical value $L_{\mathrm{C}}$ can be determined as:

$$
L_{C}=\frac{(1-D)^{2} R T}{2}
$$

When $L<L_{\mathrm{c}}$, the circuit operates in DCM, otherwise, it operates in CCM. $L_{\mathrm{c}}=556 \mu \mathrm{H}$ can be achieved by substituting the circuit parameters into Eq. (27). In order to investigate the effect of the inductance $L$ on the stability of the converter, the bifurcation diagram and Lyapunov exponent diagram with $L$ as bifurcation parameter can be drawn under the condition of $T=333.33 \mu \mathrm{s}, E=16 \mathrm{~V}, C=222$ $\mu \mathrm{F}, \mathrm{V}_{\mathrm{o}}=25 \mathrm{~V}, R=12.5 \Omega$ and $k=0.08$, which are respectively shown in Figs. 8 and 9. It can been seen that the critical stable value of $L$ is approximately $50 \mu \mathrm{H}$, and when $L$ is smaller than the value, the system will be through stable 1T orbits, stable $2 \mathrm{~T}$ orbits, stable $4 \mathrm{~T}$ orbits as $L$ decreases, and eventually approaches chaos. In Fig. 10 is shown the phase portrait around the critical of $L$.

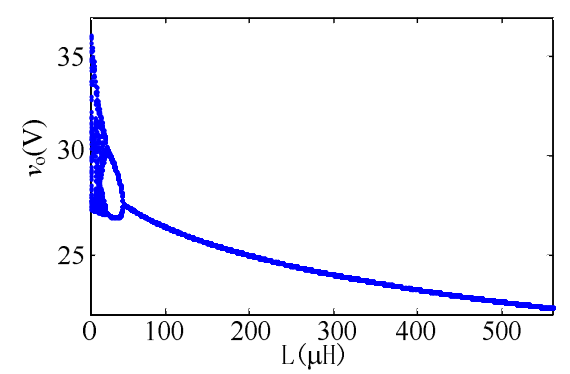

Fig. 8. Bifurcation diagram with the variation of $L$

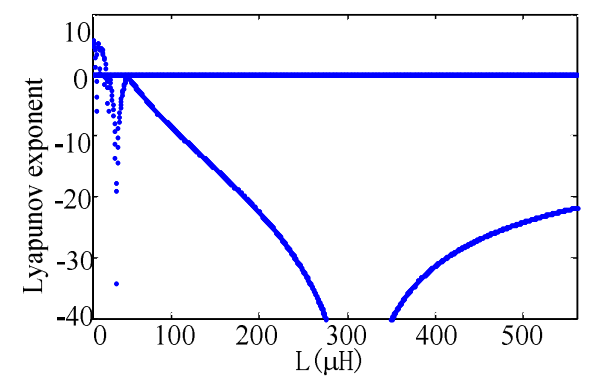

Fig. 9. Lyapunov exponent with the variation of $L$ 


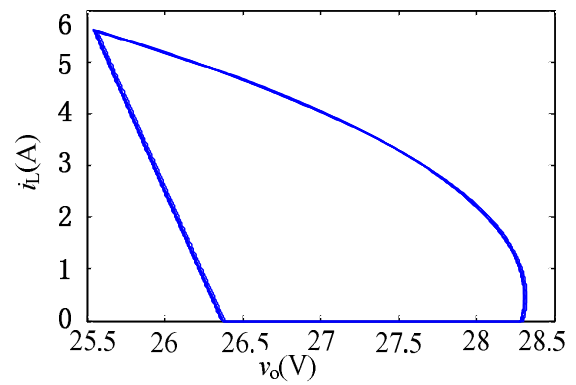

Fig. 10. Phase portraits of sampled $i_{\mathrm{L}}$ versus $v_{\mathrm{o}}$ for $L=50 \mu \mathrm{H}$.

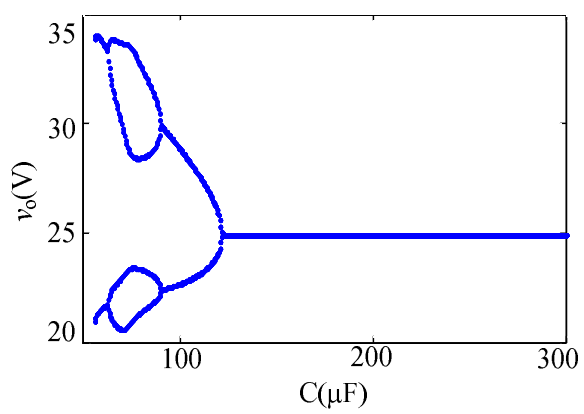

Fig. 11. Bifurcation diagram with the variation of $C$

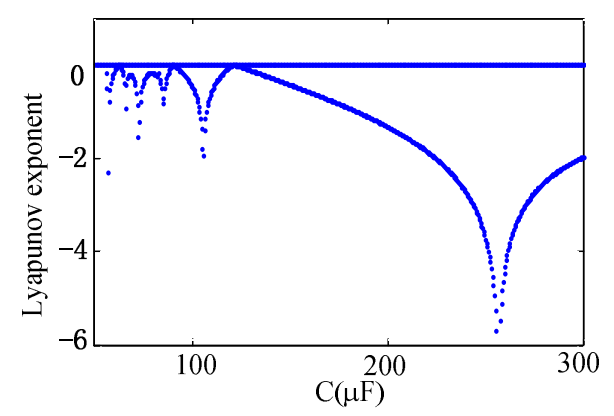

Fig. 12. Lyapunov exponent with the variation of $C$

\subsection{Effect of capacitance $C$ on system stability}

The capacitor $C$ is used to filter the output voltage to reduce the ripple. The circuit parameters are given as follows: $T=333.33 \mu \mathrm{s}, L=208 \mu \mathrm{H}, E=16 \mathrm{~V}, \mathrm{~V}_{\mathrm{o}}=25 \mathrm{~V}, R=12.5 \Omega$ and $k=0.08$. In order to investigate the relationship between $C$ and the stability of the converter, the bifurcation diagram and Lyapunov exponent diagram with $C$ as bifurcation parameter can be made with the other parameters being invariant, which are respectively shown in Figs. 11 and 12. From them, it can be seen that capacitance has a great effect on the stability of the converter, namely, the smaller the capacitance value, the poorer the stability of the converter, and the critical value is $122 \mu \mathrm{F}$, the circuit will be stable when $C \geq 122 \mu \mathrm{F}$. With the decrease of the value of the capacitor, the system will be through stable $1 \mathrm{~T}$ orbits, stable $2 \mathrm{~T}$ orbits, stable $4 \mathrm{~T}$ orbits, and eventually to chaos. The phase portrait around the critical of $C$ is shown in Fig. 13.

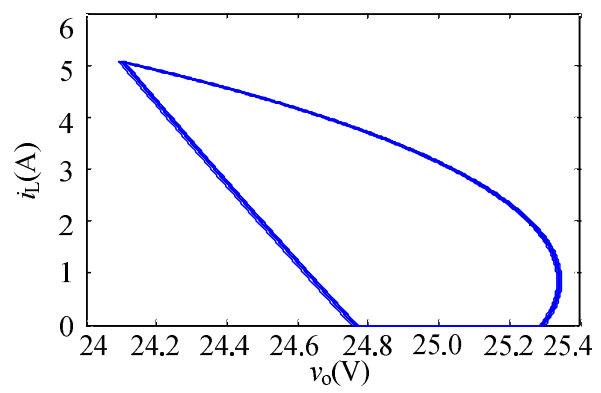

Fig. 13. Phase portraits of sampled $i_{\mathrm{L}}$ versus $v_{\mathrm{o}}$ for $C=$ $122 \mu \mathrm{F}$.

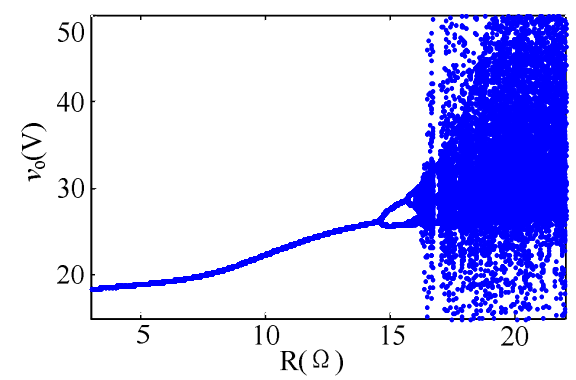

Fig.14. Bifurcation diagram with the variation of $R$

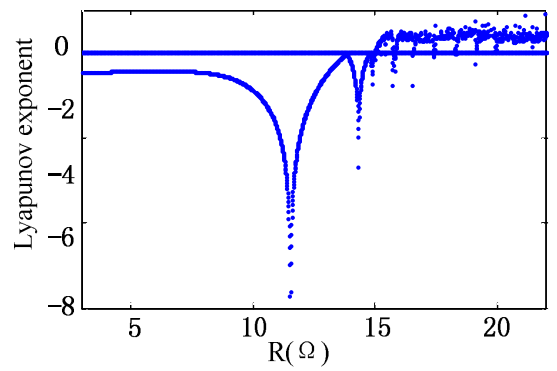

Fig. 15. Lyapunov exponent with the variation of $R$

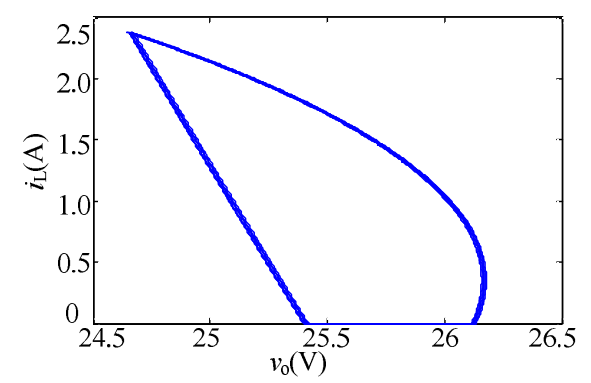

Fig. 16. Phase portraits of sampled $i_{\mathrm{L}}$ versus $v_{\mathrm{o}}$ for $R=14.5 \Omega$.

\subsection{Effect of load $R$ on system stability}

In order to study the effect of the load on the system stability, according to the stability criterion Eq. (19), the critical value of the load resistance has been found as $14.5 \Omega$ by solving Eq. (23) with the other parameters being invariant, and the bifurcation diagram has been also obtained, as shown in Fig. 14, along with the corresponding Lyapunov exponent diagram as shown in Fig. 15. From 
them, it can be seen that the effect of the load resistance on the system stability is: the bigger the resistance value, the poorer the system stability and the circuit will be stable when $R \leq 14.5 \Omega$ and the phase portrait around the critical of $R$ is shown in Fig. 16.

\section{Conclusions}

The precise iterative map of the voltage-fed buck-boost converter which is operating in DCM is proposed in this paper. Based on the map, the effect of the main circuit parameters on the system stability has been investigated, and the results obtained show that they have a great influence on the stability of the system, and the general trend is that the increase of $k, E$ or $R$, or the decrease of $L$ or $C$ will make the system stability poorer, and that all the parameters have a critical value, and when they are greater or less than the value, the system will go through perioddoubling bifurcation and eventually approaches chaos. It can be determined that the critical values of $k, E, R, L$ and $C$ are respectively $0.1569,32.2 \mathrm{~V}, 14.5 \Omega, 50 \mu \mathrm{H}$ and $122 \mu \mathrm{F}$, with the other parameters being constant.

\section{Acknowledgment}

This study was funded by Key Laboratory of Guangxi Electric Power System Optimization and Energy Saving Technology (Guangxi University) and Nanning Science Research and Technology Development Project of China (Grant number: 20121025)

\section{References:}

[1] A. Kavitha and G. Uma, "Experimental Verification of Hopf Bifurcation in DC-DC Luo Converter," Power Electronics, IEEE Trans, vol. 23, pp. 28782883, Jan.2008.

[2] C. K. Tse, S. C. Fung and M. W. Kwan, "Experimental confirmation of chaos in a current-programmed Cuk converter," Circuits and Systems I: Fundamental Theory and Applications, IEEE Trans, vol. 43, pp. 605-608, Jul.1996.

[3] C. K. Tse, "Flip bifurcation and chaos in three-state boost switching regulators," Circuits and Systems I: Fundamental Theory and Applications, IEEE Trans, vol. 41, pp. 16-23, Jan.1994.

[4] Wang Jingmei and Bi Chuang, "Effect of leakage inductance on bifurcation and chaos in the voltagefed forward converter," in Mechatronics and Automation ICMA 2009 Conference, Changchun, Aug. 2009.

[5] Boumediene Allaoua and Abdellah Laoufi, "Application of a Robust Fuzzy Sliding Mode Controller
Synthesis on a Buck-Boost DC-DC Converter Power Supply for an Electric Vehicle Propulsion System," Journal of Electrical Engineering \& Technology, vol. 6, pp. 67-75, Jan.2011.

[6] Xu Cuidong, K. W. E. Cheng and Z. H. Shi, "Nonlinear phenomena of the buck-boost converter analyzed by storage energy," in Power Electronics Systems and Applications PESA2009 3rd International Conference, Hong Kong, May.2009.

[7] Gong Ren-Xi, Xie Ling-Ling and Wang Kuang, "A Novel Modeling Method of Nonideal Buck-Boost Converter in DCM," in Information and Computing ICIC 2010 Third International Conference, Wuxi, Jiang Su, Jun.2010.

[8] Anbukumar Kavitha and Govindarajan Uma, "Resonant Parametric Perturbation Method to Control Chaos in Current Mode Controlled DC-DC BuckBoost Converter," Journal of Electrical Engineering \& Technology, vol. 5, pp. 171-178, Mar.2010.

[9] Zhang Bo and Qu Ying, "study on the experiment and the precise discerete model of bifurcation and chaos for buck DC/DC converter," Proeedings of the CSEE, pp. 102-106, 2003.

[10] Biswarup Basak and Sukanya Parui, "Exploration of Bifurcation and Chaos in Buck Converter Supplied From a Rectifier," Power Electronics, IEEE Trans, vol. 25, pp. 1556-1564, Jun.2010.

[11] Wang Xuemei, Zhang Bo and Qiu Dongyuan, "The Quantitative Characterization of Symbolic Series of a Boost Converter," Power Electronics, IEEE Trans, vol. 26, pp. 2101-2105, Aug.2011.

[12] Xie Ling-Ling, Gong Ren-Xi and Zhuo Hao-Ze, "Investigation of tangent bifurcation in voltage mode controlled DCM boost converters," ACTA PHYSICA SINICA, vol. 61, Mar. 2012.

[13] Lihong H, Meimei J, Guangyan S. "Chaos control for the Buck-Boost converter under current-mode control", in Third International Workshop on Advanced Computational Intelligence Suzhou, Jiangsu, Aug. 2010.

[14] Ling-Ling Xie, Ren-Xi Gong and Hao-Ze Zhuo, "Investigation of the Mechanism of Period-doubling Bifurcation in Voltage Mode Controlled Buck-Boost Converter," Journal of Electrical Engineering \& Technology, vol. 6, pp. 519-526, Jul.2011.

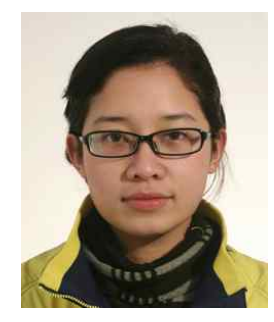

Zhao-He Feng was born in Guangxi Province, China, in 1986. She received the B.S. degrees in Automation from Beifang University of Nationalities, Yinchuan, China, in 2010. She is currently working on her Ph.D. in electric power system and automation in Guangxi University, Nanning, China Her main research interests include nonlinear dynamics, 
stability analysis of hybrid systems, and application of power electronics in renewable energies.

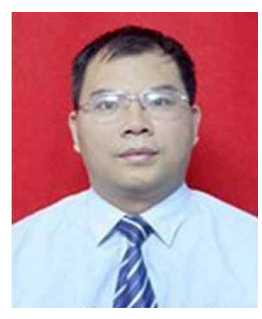

Ren-Xi Gong was born in Guilin of Guangxi Province in September 1962. From August 1990 to March 1993, he was a graduate student majoring in Semiconductor Devices and Microelectronics at Xidian University. $\mathrm{He}$ received his M.S. degree in March 1993. From 1994 to 1997, he worked as an assistant at Guangxi University, and from February 1998 to January 2002, he worked on his Ph.D degree in microelectronics and solid-state electronics at Xidian University. $\mathrm{He}$ is currently a professor at Electrical Engineering School of Guangxi University. His present research interests are intelligent detection technology, power electronics and their applications.

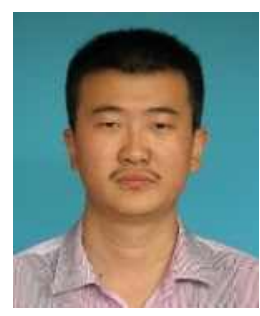

Qing-Yu Wang was born in Guizhou Province, China, in 1988. He received his B.S. degrees in Electronic Science and Technology from Guangxi University, Nanning, China in 2011. He is currently working on his Ph.D. in electric power system and automation in the same university. His research interests in nonlinear dynamics, optimization, and power electronics. 